\title{
Bistability and simultaneous mode actuation in electrostatically actuated initially curved coupled micro beams
}

\author{
Lior Medina $^{1} \quad$ Ashwin A. Seshia ${ }^{1}$ \\ ${ }^{1}$ Nanoscience Centre, Department of Engineering, University of Cambridge, Cambridge, CB3 OFF, United \\ Kingdom
}

\begin{abstract}
The symmetric snap-through response of a bistable structure, composed from two beams, that are coupled via a rigid truss at their midpoint, is studied when subjected to a distributed electrostatic load. Both beams are double clamped and initially curved. The analysis is based on a reduced order (RO) model, resulting from Galerkin's decomposition. For the base functions, symmetric buckling modes are used for either beam. The results of the RO model are compared with results obtained via finite differences (FD) solutions, to validate the approximation to the original differential formulation, and a finite element (FE) model. Specifically, FE analysis was used as a reference under "mechanical" displacementindependent load, facilitating the usage of solutions extracted via FD for validation of the model under electrostatic, displacement-dependent, load. All solutions employed the usage of the arc-length "Riks" method to accommodate swerving equilibrium paths. To enable a broader approach, the two beams may have different initial elevations. The study indicates that a model with at least three degrees of freedom (DOF) is needed to depict an equilibrium path, for either load. For reliably quantitative equilibrium curves, a model with a minimum of five DOF was found to be necessary. The presented results also indicate that a double curved beam structure can attain actuation of several modes simultaneously, while demonstrating snap-through at reasonable voltages. In so doing, the model suggests that such a construct can be feasible for usage in various applications.
\end{abstract}

Keywords:

Snap-through, Electrostatic loading, High buckling modes, Riks method, MEMS/NEMS 


\section{Introduction}

Curved beams and plates, loaded by either distributed or concentrated loads may have bistable properties, where a beam would possess two stable equilibria under the same loading [1]. Such a property was shown to be useful and favourable in a large variety of applications, especially in the micro scale. Among such applications are shock sensors $[2,3]$ threshold accelerometers $[4,5,6]$, logical elements $[7,8,9]$, non-volatile mechanical memories $[10,11$, 12, 13], switches [14, 15], and energy harvesters $[16,17,18]$. As a result, research on a structure which at first glance may seem as a classic construct, and as such was heavily studied in the past $[19,20]$, was reinvigorated due to the addition of nonlinear loads and phenomena, such as a pull-in response, not encountered before [21]. Among them one is able to mention the effect of magnetic loads [22], electrostatic loads [23, 24, 25, 26], the combined effect of direct and alternating currents which can induce bistability [27], and Casimir forces [28], just to name a few.

Lately, the focus of bistable structures is starting to shift to coupled constructs, such as two curved beams, connected at their respective midpoints via a truss, or a rigid rod. First mentioned in [29], where it was found that a double beam structure can lessen the effect of symmetry breaking related bifurcations [19], provided that the two beams are identical. Later, it was discovered that such a structure cannot be bistable if the geometries of either

beam are not carefully selected. In addition, it was also found that the longer the bridge (truss) between the two beams, the easier it is to overcome the second mode, which is alternatively the first asymmetric mode, at the cost of increasing the loading curve of the resulting structure [30]. The result of these conclusions and observations reverberated to new studies, where a symmetric response was a favourable outcome [31], and when asymmetric bifurcations were found to be an obstacle to various phenomena such as latching [32, 33, 34, 35] and dynamic release [36].

As a result, more and more studies, where double beam structures, or similarly "double V beam" structures, have been the subject of intensive research of late. In [37], a mechanical switch comprised of two double beams and a V-beam actuator, was developed to enable a 
two bit mechanism using thermal actuation. Later, a bistability criterion was formulated and validated for a double V-beam mechanism [38], followed by an upgrade presented in [39]. Since a V-beam structure was being repeatedly used, design criteria for a V-beam actuator have been formulated [40]. Next, by using finite element (FE) analysis, a compliant V-shaped bistable mechanism has been explored for usage in space based applications. The analysis indicated that such a structure could be used in thermal actuators, switches, latches, force sensors, launch docks, camera covers and deployable structures, when used in larger scales [14]. In light of this growing interest, a bistability condition was later found via analytical techniques for a double beam structure with different initial curvatures, under "mechanical", displacement-independent, load [41]. In doing so, the nonlinear behaviour of such a structure was better understood, proving that bistability depends on the elevation to thickness ratios of both beams, and that bistability of the structure as a whole is not guaranteed if just one of the beams is bistable. Lastly, a double beam bistable device was shown, where actuation was introduced via a moment based input, facilitating snap-through response using a single driving source [42].

From the above review it is therefore clear that there is significant research interest in the engineering and design of such structures. However, the behaviour of the double beam construct was not studied under the effect of a distributed, nonlinear, displacement-dependent, electrostatic load, especially when considering that the two beams do not share identical curvatures. To fill that gap, we expand previous studies that were solved for mechanical loads, facilitating the usage of such a structure for electrostatic based applications. In addition, we also take a broader approach, observing the behaviour of such a structure when the two beams are not identical, while also taking a closer look at the interaction between the two beams. To make it so, we formulate a reduced order ( $\mathrm{RO})$ model, derived from the coupled shallow beam equations, present a case study, and provide validation of the said model. The end result of the presented study therefore lies in providing a trustworthy RO model, that could be used for design and conception of electrostatically actuated, coupled, double micro beams based structures. 

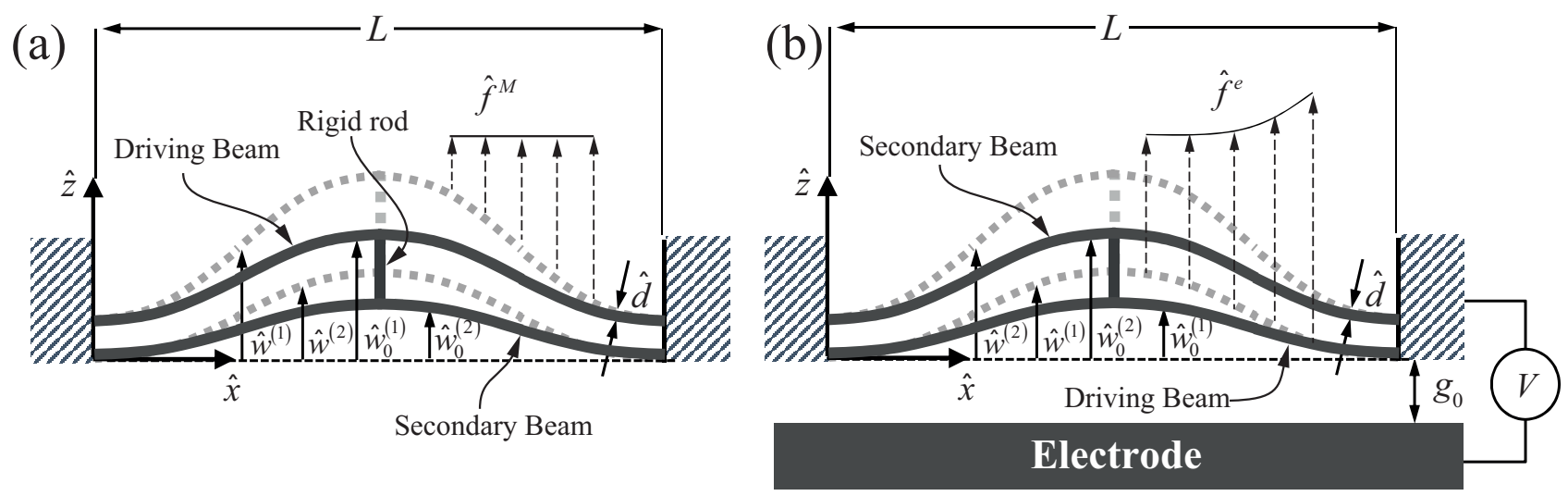

Figure 1: Model of initially curved, double clamped, coupled beams. Both beams have thickness $\hat{d}$ and different initial configurations $\hat{w}_{0}^{(1)}$ and $\hat{w}_{0}^{(2)}$. The beams are coupled at their respective midpoint (i.e., at $\hat{x}=L / 2$ ) via a rigid rod. The structure is shown here under two loads: (a) Distributed mechanical load $\left(\hat{f}^{M}\right)$ bearing on the upper beam, designated as the "Driving Beam" or alternatively Beam \#1, causing the movement of the lower beam, designated as the "Secondary Beam", or Beam \#2. (b) Distributed electrostatic load $\hat{f}^{e}$, acting on the lower beam, now redefined as the "Driving Beam", via an electrode located at a distance $g_{0}$. The "Secondary Beam" is located above it. The dashed line corresponds to the deformed configuration of either beam, designated as $\hat{w}^{(1)}$ and $\hat{w}^{(2)}$ for Beam \#1 and $\# 2$, respectively. Positive directions of the beams deflections and of the loadings are shown.

\section{Formulation}

We consider two flexible initially curved, double clamped prismatic micro beams of length $L$, having a rectangular cross-section of width $b$ and thickness $\hat{d}$, connected via a rigid rod at their respective midpoints, as shown in Fig. 1. The beams are made of homogeneous, isotropic, linearly elastic material, with Young's modulus $E$ and Poisson's ratio $\nu$. Since the width $b$ of both beams is typically larger than their thickness $\hat{d}$, an effective (plane strain) modulus of elasticity $\tilde{E}=E /\left(1-\nu^{2}\right)$ is used. The initial shape of each beam is described by the functions $\hat{w}_{0}^{(i)}(\hat{x})=\hat{h}_{0}^{(i)} z_{0}(\hat{x})$, where $\hat{h}_{0}^{(i)}$ is the initial elevation of the $i^{\text {th }}$ beam $(i=1,2)$ central point above its ends, and $z_{0}(\hat{x})$ is a non-dimensional function with a normalized maximum of $\max _{\hat{x} \in[0, L]}\left[z_{0}(\hat{x})\right]=1$. The main assumption of the model is that one of the beams, designated as the "Driving Beam" (alternately, Beam \#1), encounters the load, while the other beam, designated as the "Secondary Beam" (alternately, Beam \#2), reacts to the movement of the former via a reaction force, emanating from the rigid rod. For that reason, when a "mechanical" load is present, the Driving Beam is the upper one, as seen in Fig. 1(a), whereas for an electrostatic load, the Driving Beam is the lower one. For 
the latter, the load is provided by an electrode located at a distance $g_{0}$ (the gap) from the lower beam ends. Note, that due to the rigid rod, the two beams move together with equal displacement at their midpoint.

We assume that $\hat{d} \ll L, \hat{h}_{0}^{(i)} \ll L$ and that either beam undergoes moderately large deflections compared to its thickness to induce axial stress, changing each beam stiffness in a nonlinear fashion. Under these assumptions, the beams behaviour is described in the framework of the Euler-Bernoulli beam theory, combined with the shallow arch approximation. As such, we take into account the stretching of either beam midplane and the axial force that emanates from it due to displacement, while neglecting rotation as a second order effect. The result is a description of a single beam via two equations [25, 43, 44, 45]. However, since the current model is comprised of two beams, which could also have different curvatures, the formulation of a single beam is therefore used twice, while acknowledging that each beam faces a different loading scheme. The result is a system of four equations, describing the behaviour of the structure $(i=1,2)$

$$
\begin{array}{r}
\left.\frac{\mathrm{d}}{\mathrm{d} \hat{x}}\left(\tilde{E} A\left(\frac{\mathrm{d} \hat{u}^{(i)}}{\mathrm{d} \hat{x}}+\frac{1}{2}\left(\frac{\mathrm{d} \hat{w}^{(i)}}{\mathrm{d} \hat{x}}\right)^{2}-\frac{1}{2}\left(\frac{\mathrm{d} \hat{w}_{0}^{(i)}}{\mathrm{d} \hat{x}}\right)\right)^{2}\right)\right) \\
\tilde{E} I_{y y}\left(\frac{\mathrm{d}^{4} \hat{w}^{(i)}}{\mathrm{d} \hat{x}^{4}}-\frac{\mathrm{d}^{4} \hat{w}_{0}^{(i)}}{\mathrm{d} \hat{x}^{4}}\right)-\frac{\mathrm{d}}{\mathrm{d} \hat{x}}\left(\frac{\mathrm{d} \hat{w}^{(i)}}{\mathrm{d} \hat{x}}\left(\tilde{E} A\left(\frac{\mathrm{d} \hat{u}^{(i)}}{\mathrm{d} \hat{x}}+\frac{1}{2}\left(\frac{\mathrm{d} \hat{w}^{(i)}}{\mathrm{d} \hat{x}}\right)^{2}-\frac{1}{2}\left(\frac{\mathrm{d} \hat{w}_{0}^{(i)}}{\mathrm{d} \hat{x}}\right)\right)\right)+\hat{F}^{(i)}\right)
\end{array}
$$

where $\hat{u}, A$ and $I_{y y}$ are the axial displacement, cross-section area and second moment of inertia with respect to the $\hat{y}$ axis. To distinguish between the derivative and the beams thickness, we represent the former with a non-slanted " $d$ ". The above equations are accompanied by the following boundary conditions, for each beam

$$
\hat{u}^{(i)}(0)=\hat{u}^{(i)}(L)=0 \quad \hat{w}^{(i)}(0)=\hat{w}^{(i)}(L)=\left.0 \quad \frac{\mathrm{d} \hat{w}^{(i)}}{\mathrm{d} \hat{x}}\right|_{x=0}=\left.\frac{\mathrm{d} \hat{w}^{(i)}}{\mathrm{d} \hat{x}}\right|_{x=L}=0
$$

as well as a compatibility condition, stating that the displacement of the two beams is equal 
at the midpoint

$$
\hat{w}^{(1)}\left(\frac{L}{2}\right)-\hat{w}_{0}^{(1)}\left(\frac{L}{2}\right)=\hat{w}^{(2)}\left(\frac{L}{2}\right)-\hat{w}_{0}^{(2)}\left(\frac{L}{2}\right)
$$

where ()$^{(i)}$ signifies that the term belongs to beam $i=1,2$. In addition, $\hat{F}^{(i)}$ is the loading that beam $i$ encounters, given by

$$
\hat{F}^{(i)}=\delta_{1 i} \hat{f}+(-1)^{i} \hat{F}_{R} \hat{\delta}\left(\hat{x}-\frac{L}{2}\right)
$$

with $\hat{f}$ representing the distributed lateral load bearing on the Driving Beam, which can be mechanical $\hat{f}^{M}$, or electrostatic $\hat{f}^{e}$. Note, that although the latter could take into account the effect of fringing fields, and while their existence does decrease the voltage of the equilibrium curve [24, 25], it was previously found that it does not alter results concerning the location of the limit points, or the presence of bistability [46]. Taking that into account, we use, for the sake of simplicity, the following parallel capacitor plate approximation

$$
\hat{f}^{e}=\frac{\epsilon_{0} b V^{2}}{2\left(g_{0}+\hat{w}^{(1)}(\hat{x})\right)^{2}}
$$

where $\epsilon_{0}=8.854 \times 10^{-12} \mathrm{~F} / \mathrm{m}$ is the permittivity of the free space, and $V$ is the voltage difference between the Driving beam and the electrode. Note that the sign is consistent with Fig. 1. In addition, $\hat{F}_{R}$ represents the reaction force, limited to act only at the midpoint (i.e., $\hat{x}=L / 2$ ) via a Dirac delta function, $\hat{\delta}$. Also, $\delta_{1 i}$ signifies the Kronecker delta, stating that the aforementioned distributed load exists only for Beam \#1, while the reaction force, being relevant for both, has an opposite sign for Beam \#2.

For convenience, we re-write Eqs. (1), (2) in a non-dimensional form for the $i^{\text {th }}$ beam

$$
\begin{array}{r}
\left(2\left(u^{(i)}\right)^{\prime}+\left(\left(w^{(i)}\right)^{\prime}\right)^{2}-\left(\left(w_{0}^{(i)}\right)^{\prime}\right)^{2}\right)^{\prime}=0 \\
\left(w^{(i)}\right)^{I V}-\left(w_{0}^{(i)}\right)^{I V}-\alpha\left(\left(w^{(i)}\right)^{\prime}\left(\left(2\left(u^{(i)}\right)^{\prime}+\left(\left(w^{(i)}\right)^{\prime}\right)^{2}-\left(\left(w_{0}^{(i)}\right)^{\prime}\right)^{2}\right)\right)\right)^{\prime}+F^{(i)}
\end{array}
$$


Table 1: Non-dimensional quantities used for mechanical loading.

\begin{tabular}{|l|l|}
\hline$x \triangleq \hat{x} / L$ & axial coordinate \\
\hline$\delta \triangleq \hat{\delta} L$ & Non-dimensional Dirac delta function \\
\hline$u^{(i)} \triangleq \hat{u} L / \hat{d}^{2}$ & $i^{\text {th }}$ beam axial displacement \\
\hline$w^{(i)} \triangleq \hat{w}^{(i)} / \hat{d}, w_{0}^{(i)} \triangleq \hat{w}_{0}^{(i)} / \hat{d}$ & $i^{\text {th }}$ beam config./initial config. \\
\hline$h_{0}^{(i)} \triangleq \hat{h}_{0}^{(i)} / \hat{d}$ & $i^{\text {th }}$ beam initial elevation at the midpoint \\
\hline$\alpha^{M} \triangleq A \hat{d}^{2} /\left(2 I_{y y}\right)=6$ & Stretching parameter \\
\hline$F_{R}^{M} \triangleq \hat{F}_{R}^{e} L^{3} /\left(\tilde{E} I_{y y} \hat{d}\right)$ & Reaction force parameter \\
\hline$\beta^{M} \triangleq q L^{4} /\left(\tilde{E} I_{y y} \hat{d}\right)$ & Mechanical load parameter \\
\hline
\end{tabular}

with homogeneous boundary conditions at $x=0,1$ alongside the compatibility condition from Eq. (3), which takes the form

$$
w^{(1)}\left(\frac{1}{2}\right)-w_{0}^{(1)}\left(\frac{1}{2}\right)=w^{(2)}\left(\frac{1}{2}\right)-w_{0}^{(2)}\left(\frac{1}{2}\right)
$$

where ( $)^{\prime}$ and ( $)^{I V}$ denote first and fourth derivatives, respectively, with respect to the non-dimensional coordinate $x, 0 \leq x \leq 1$. The non-dimensional equivalent of Eqs. (4), (5) are expressed by

$$
F^{(i)}=\delta_{1 i} f+(-1)^{i} F_{R} \delta\left(x-\frac{1}{2}\right) \quad f^{M}=\beta^{M} \quad f^{e}=\frac{\beta}{\left(1+w^{(1)}(x)\right)^{2}}
$$

with $f$ being mechanical or electrostatic load (i.e., $f^{M}$ or $f^{e}$, respectively). The nondimensional quantities used in Eqs. (6)-(10) are defined in Tables 1 \& 2 for mechanical and electrostatic loads, respectively. Notice that per the definitions, the stretching and reaction force parameters (i.e., $\alpha$ and $F_{R}$ ) change according to the load.

In accordance with Eq. (6), the axial force along beam $i$ is constant. Therefore, Eqs. (6), (7) can be reduced to the following single non-dimensional equation for the $i^{\text {th }}$ beam (e.g., [45] for a single mechanically loaded beam). Upon doing so, and substituting the load 
Table 2: Non-dimensional quantities used for electrostatic loading.

\begin{tabular}{|l|l|}
\hline$u^{(i)} \triangleq \hat{u} L / g_{0}^{2}$ & $i^{\text {th }}$ beam axial displacement \\
\hline$w^{(i)} \triangleq \hat{w}^{(i)} / g_{0}, w_{0}^{(i)} \triangleq \hat{w}_{0}^{(i)} / g_{0}$ & $i^{\text {th }}$ beam config./initial config. \\
\hline$h_{0}^{(i)} \triangleq \hat{h}_{0}^{(i)} / g_{0}$ & $i^{\text {th }}$ beam initial elevation at the midpoint \\
\hline$d \triangleq \hat{d} / g_{0}$ & Thickness \\
\hline$\alpha^{e} \triangleq A g_{0}^{2} /\left(2 I_{y y}\right)=6 / d^{2}$ & Stretching parameter \\
\hline$F_{R}^{e} \triangleq \hat{F}_{R} L^{3} /\left(\tilde{E} I_{y y} g_{0}\right)$ & Reaction force parameter \\
\hline$\beta \triangleq \epsilon_{0} b V^{2} L^{4} /\left(2 \tilde{E} I_{y y} g_{0}^{3}\right)$ & Voltage parameter \\
\hline
\end{tabular}

expression, Eq. (9), one receives the following equation for beam $i$

$$
\left(w^{(i)}\right)^{I V}-\left(w_{0}^{(i)}\right)^{I V}-\alpha\left(\int_{0}^{1}\left(\left(\left(w^{(i)}\right)\right)^{2}-\left(\left(w_{0}^{(i)}\right)^{\prime}\right)^{2}\right) d x\right)\left(w_{0}^{(i)}\right)^{\prime \prime}+\delta_{1 i} f+(-1)^{i} F_{R} \delta\left(x-\frac{1}{2}\right)=0
$$

effectively reducing the number of equations from four, to two.

Observing Eq. (10), one is also able to note that the location of Beam \#2, with respect to Beam \#1 does not play a role. The reason is the nature of the connection between the two beams, which is rigid, causing the reaction force to be deflection-independent. Therefore, $w^{(2)}$ can be located at any point, as long it is below $w^{(1)}$ under a mechanical load, and vice versa when electrostatic load is brought to bear.

\subsection{Reduced order model}

In order to analyse the double beam response, a reduced order (RO) model based on Galerkin's decomposition, is constructed. Using the observations regarding the nature of the coupling between the beams, the approximation of either one can be constructed using the same shape functions (i.e., $\varphi_{j}^{(1)}(x) \equiv \varphi_{j}^{(2)}(x)=\varphi_{j}(x)$ ), without adding a constant off set to one of the them. As a result, we use the following series to approximate the deformed shape of the $i^{\text {th }}$ beam $\left(w^{(i)}(x)\right)$, and its corresponding initial shape $\left(w_{0}^{(i)}(x)\right)$

$$
w^{(i)}(x) \approx \sum_{j=1}^{n} q_{j}^{(i)} \varphi_{j}(x) \quad w_{0}^{(i)}(x)=\sum_{j=1}^{n} q_{0 j}^{(i)} \varphi_{j}(x)
$$


where $\varphi_{j}$ represents the $j^{\text {th }}$ base function of either beam. Although many formulations could be given to fill the role of the base functions, such as undamped or flexural vibrations modes [24, 47, 48], we chose to use a double clamped buckling eigenmodes of a straight beam. The reason behind this choice emanates from the nature of the current study, which focuses on the static behaviour of the structure, where such eigenfunctions are closer in terms of shape when compared to vibrations based eigenfunctions [49]. The eigenmodes are defined in such a way so that $\max _{x \in[0,1]}\left(\varphi_{j}(x)\right)=1$. Moreover, $n$ is the total amount of degrees of freedom (DOF)/modes, taken for the approximation. Substitution of Eq. (11) into Eq. (10), multiplication by $\varphi_{k}$ and followup integration, produces a system with $n$ coupled nonlinear algebraic equations, for either beam

$$
\mathbf{B}\left(\mathbf{q}^{(i)}-\mathbf{q}_{\mathbf{0}}^{(i)}\right)+\alpha\left(\left(\left(\mathbf{q}^{(i)}\right)^{\mathbf{T}} \mathbf{S} \mathbf{q}^{(i)}-\left(\mathbf{q}_{\mathbf{0}}^{(i)}\right)^{\mathbf{T}} \mathbf{S} \mathbf{q}_{\mathbf{0}}^{(i)}\right)\right) \mathbf{S} \mathbf{q}^{(i)}+\delta_{1 i} \mathbf{f}+(-1)^{i} \mathbf{f}_{\mathbf{R}}=0
$$

where ()$^{T}$ denotes the matrix transpose, and $\mathbf{q}^{(i)}=\left\{q_{j}^{(i)}\right\}, \mathbf{q}_{\mathbf{0}}^{(i)}=\left\{q_{0 j}^{(i)}\right\}$ are the general coordinate vectors of the deformed and initial shapes, respectively. The elements of the matrices $\mathbf{B}=\left\{b_{j k}\right\}$ and $\mathbf{S}=\left\{s_{j k}\right\}$ are associated with the bending and stretching stiffness of either beam, respectively, and are given by the expressions

$$
b_{j k}=\delta_{j k} \int_{0}^{1} \varphi_{j}^{\prime \prime} \varphi_{k}^{\prime \prime} d x \quad s_{j k}=\delta_{j k} \int_{0}^{1} \varphi_{j}^{\prime} \varphi_{k}^{\prime} d x
$$

with $\delta_{j k}$ representing the Kronecker delta, resulting from the orthogonality of the buckling eigenmodes. The elements of the generalized force vectors, $\mathbf{f}=\left\{f_{j}\right\}, \mathbf{f}_{\mathbf{R}}=\left\{f_{R_{j}}\right\}$, are associated with the lateral loading and reaction force, respectively. Since the former can represent either mechanical $\left(f_{j}=f_{j}^{M}\right)$ or electrostatic $\left(f_{j}=f_{j}^{e}\right)$ loading, substitution of $f_{j}=f_{j}^{M}$ or $f_{j}=f_{j}^{e}$ will take place, as will for the reaction force, for which $F_{R}=F_{R}^{M}$ or 
$F_{R}=F_{R}^{e}$, given by

$$
f_{j}^{M}=\beta^{M} \int_{0}^{1} \varphi_{j}(x) d x \quad f_{j}^{e}=\beta \int_{0}^{1} \frac{\varphi_{j}(x)}{\left(1+\sum_{k=1}^{n} q_{k}^{(1)} \varphi_{k}(x)\right)^{2}} d x \quad f_{R_{j}}=F_{R} \varphi_{j}\left(\frac{1}{2}\right)
$$

At this point in the derivation, it is of value to point out that for a case in which both beams have the same geometry (i.e., $w_{0}^{(1)}(x)=w_{0}^{(2)}(x)$ ), an asymmetric response will not be apparent, as noted in [29]. Since the present study takes a broader view, allowing the beams to have different elevations, it is safe to assume that twisting of the midpoint due to bifurcations may take place if certain elevations are reached [20, 23, 48]. However, as pointed out in [30], if the spacing between the two beams (i.e., $\left|w_{0}^{(1)}(0)-w_{0}^{(2)}(0)\right|=$ $\left.\left|w^{(1)}(0)-w^{(2)}(0)\right|\right)$ is large enough, then overcoming the second mode can be made possible. Since the devised model is independent of the said gap, a value can be taken to overcome symmetry breaking. Also, since the goal of the present paper is the study of bistablity behaviour, we therefore focus on the symmetric response. It is worthy to note, that even in the presence of bifurcations, a symmetric response is still present and is considered as a possible response, even more so when taking into account that bistability is a prerequisite for symmetry breaking [20, 25, 26, 34, 48].

Using the above symmetry assumption, we perform Galerkin's approximation procedure on the compatibility condition, Eq. (8). Taking into account that even numbered modes have zero deflection at the midpoint (i.e., $\varphi_{j}(1 / 2)=0$ for even numbered $j$ ), the resulting condition is reduced to the following expression

$$
\sum_{\text {For odd } j=1}^{n} q_{j}^{(1)}-h_{0}^{(1)}=\sum_{\text {For odd } j=1}^{n} q_{j}^{(2)}-h_{0}^{(2)}
$$

Equations (12), (15) complete the formulation of the RO model to be studied. However, before we delve into the study itself, it is instructive to look first at a single DOF model (for $n=1$ ), producing three equations, one for each beam, along with the compatibility 
condition, resulting in the following system

$$
\begin{array}{r}
b_{11}\left(q_{1}^{(1)}-h_{0}^{(1)}\right)+\alpha\left(\left(q_{1}^{(1)}\right)^{2}-\left(h_{0}^{(1)}\right)^{2}\right) s_{11}^{2} q_{1}^{(1)}+f-f_{R 1}=0 \\
b_{11}\left(q_{1}^{(2)}-h_{0}^{(2)}\right)+\alpha\left(\left(q_{1}^{(2)}\right)^{2}-\left(h_{0}^{(2)}\right)^{2}\right) s_{11}^{2} q_{1}^{(2)}+f_{R_{1}}=0 \\
q_{1}^{(1)}-h_{0}^{(1)}-q_{1}^{(2)}+h_{0}^{(2)}=0
\end{array}
$$

By showing the condition from Eq. (18)for $q_{1}^{(2)}$, while expressing $f_{R 1}$ from Eq. (17) in terms of $q_{1}^{(2)}$, and substituting both expressions in Eq. (16), one receives

$$
\begin{aligned}
& 2 b_{11}\left(q_{1}^{(1)}-h_{0}^{(1)}\right)+ \alpha\left(\left(\left(q_{1}^{(1)}-h_{0}^{(1)}+h_{0}^{(2)}\right)^{2}+\left(q_{1}^{(1)}\right)^{2}-\left(h_{0}^{(2)}\right)^{2}-\left(h_{0}^{(1)}\right)^{2}\right)\right. \\
&\left.q_{1}^{(1)}+\left(\left(q_{1}^{(1)}-h_{0}^{(1)}\right)^{2}+h_{0}^{(2)}\left(q_{1}^{(1)}-h_{0}^{(1)}\right)\right)\left(h_{0}^{(2)}-h_{0}^{(1)}\right)\right) s_{11}^{2}+f=0
\end{aligned}
$$

The resulting equation shows a single equation with an expanded stretching term (related to $s_{11}$ ) and a higher bending stiffness (related to $b_{11}$ ). Note that for a private case, at which the two beams are identical, $\left(h_{0}^{(1)}=h_{0}^{(2)}\right)$, one receives a double beam with twice the stiffness, which in turn requires twice the load to create a displacement, resulting in

$$
b_{11}\left(q_{1}^{(1)}-h_{1}^{(1)}\right)+\alpha\left(\left(q_{1}^{(1)}\right)^{2}-\left(h_{0}^{(1)}\right)^{2}\right) q_{1}^{(1)} s_{11}^{2}+\frac{f}{2}=0
$$

Such an outcome coincides with conclusions made in [30], there it was shown that if the two beams are identical, the load required will be twice that of a single beam.

Expanding the above realization to $n$ DOF, we first express $q_{1}^{(2)}$ from Eq. (15), and substitute it in Eq. (12) for both beams. Following that, we express the reaction force from the first row $(j=1)$ of the second beam $(i=2)$, resulting in an expression for $f_{R}$, which is substituted back to all remaining equations, i.e. $j=1 . . n$ for $i=1$ and $j=2 . . n$ for $i=2$. Realizing that the general coordinates of the second beam are essentially additional DOF in the double beam structure, we perform a change of variables, redefining $q_{1}^{(1)} . . q_{n}^{(1)}$ as $q_{1} . . q_{n}$, and $q_{2}^{(2)} \ldots q_{n}^{(2)}$ as $q_{n+1} \ldots q_{2 n-1}$. The result of this procedure is a new system of equations 
composed of $2 n-1$ DOF.

\section{Results \& discussion}

Having completed the formulation, we now turn to the case study and validation of the model. Since previous instances have shown that bistable structures can possess complicated behaviour, resulting in equilibrium curves that can swerve and twist in a complex pattern $[34,50]$, we turn to calculation via the "Riks" (a.k.a. the arc-length) method $[50,51,52]$.

\subsection{Mechanical load}

Validation of the model under mechanical load is carried out in the following sequence. We first analyse the convergence of the model against a ten DOF model, by substituting $\alpha=\alpha^{M}$ and $\mathbf{f}=\mathbf{f}^{M}$, for a range of $n=1 . .10$, in Eqs. (11), (12), (15), allowing us to witness the ability of the model to converge, while observing its trend, with respect to two different configurations. Following that, we move on to a comparison against an FD solution of Eqs. (6), (7) with $f=f^{M}, F_{R}=F_{R}^{M}, \alpha=\alpha^{M}$, which will verify the RO approximation to its original differential model. Finally, we compare the model against an FE model, which serves as the reference for this type of loading. Although many convergence parameters could be selected, we chose to track the location and load of the snap-through point, since such tracking can focus on the ability of the model to foretell bistability [53]. At this stage it is worth mentioning which beam is being tracked. To make such a decision, one must consider the difference in loading schemes. As seen in Fig. 1(a) for mechanical load and Fig. 1(b) for electrostatic load, the Secondary Beam experiences a concentrated (mechanical) load at its centre, whilst the Driving Beam has a distributed (mechanical or electrostatic) lateral load, as well as a concentrated force, emanating from the truss. As a result, it is possible to deduce that of the two, the Driving Beam faces the complex loading, which has the potential to produce complicated responses. Taking that into account has brought us to conclude that for the purpose of the case study and model validation, we will focus on the Driving Beam. The interaction of both beams is addressed later, after validation of the model is completed. 
The FE analysis was carried out using ABAQUS with B21H beam elements, and T2D2 truss elements for the rigid rod [54]. For the latter, axial rigidity was defined to be $E A=1000$ MPa. To determine how many elements are required, a convergence study, employing the arc-length method, was carried out for a double beam structure with $\hat{d}=2 \mu \mathrm{m}, \hat{h}_{0}^{(1)}=6 \mu \mathrm{m}$, $\hat{h}_{0}^{(2)}=3 \mu \mathrm{m}, L=1000 \mu \mathrm{m}, E=169 \mathrm{GPa}$ and $\nu=0.24$, and varying number of elements, ranging from 5 to 2010, encompassing both beams, as well as the rigid rod. The study showed that with respect to the most refined mesh, a 1005 elements model was able to attain errors of $\approx 0.053 \%$ and $\approx 0.002 \%$ for the snap-through location and load, respectively. Since the new structure depends on the ratio between the two beams as well [41], another configuration was selected, defined by $\hat{h}_{0}^{(1)}=8 \mu \mathrm{m}, \hat{h}_{0}^{(2)}=5.6 \mu \mathrm{m}$. Note that all other parameters remained the same. The resulting errors were found to be $\approx 0.078 \%$ and $\approx 0.0004 \%$ for the snap-through location and load, respectively, for 1005 elements, when compared to the 2010 elements based mesh. Having done so, we henceforth used a mesh composed of 1005 elements as the reference for the mechanical load analysis. As a final verification of the model, the first configuration was altered yet again, with a different beam spacing (i.e., $\left|w^{(1)}(0)-w^{(2)}(0)\right|$ ), to ensure our model assumption, relating to the distance between the beams, holds. While the convergence was carried out for a gap of $10 \mu \mathrm{m}$, the refined mesh was calculated again for a gap of $15 \mu \mathrm{m}$. Comparing the two meshes has produced an error of $0.009 \%$ for the snapthrough location with respect to the original configuration. To conclude, although different gaps were used, it did not hamper the overlap of both curves, resulting in a negligible error. Therefore, the modelling assumption made in Sec. 2.1, which simplified the model, bears merit.

As for the FD based solution, the full formulation given in Eqs. (6), (7) was decomposed for both axial and lateral displacements using the central differences approximation [55], for either beam. Note that for the FD, the arc-length method is used to find the coordinates along the beam, where as for the RO model it is used to find the general coordinates corresponding to the modes. Taking that into account, the number of segments along the beams has to be even, so that there would be a node at the midpoint, where the two beams are 
(a)

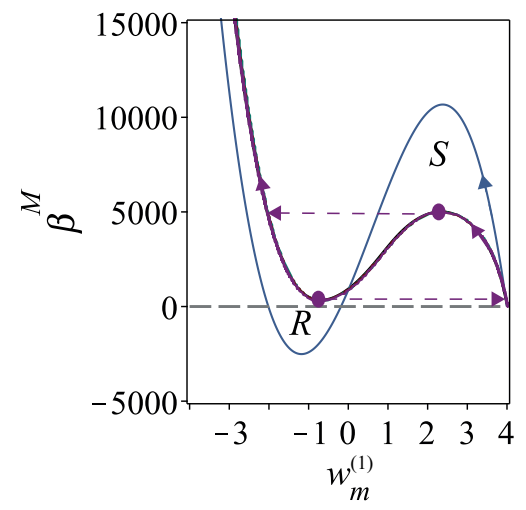

(c)

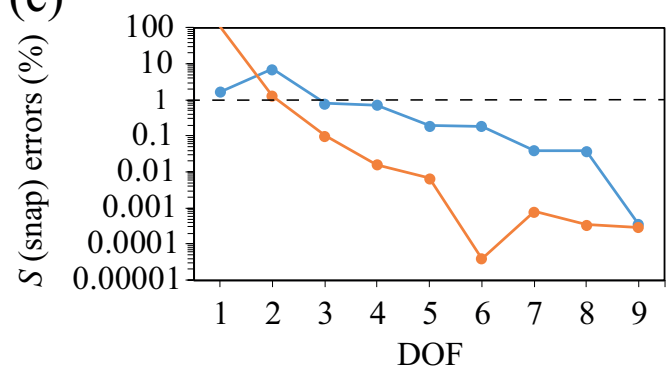

(e)

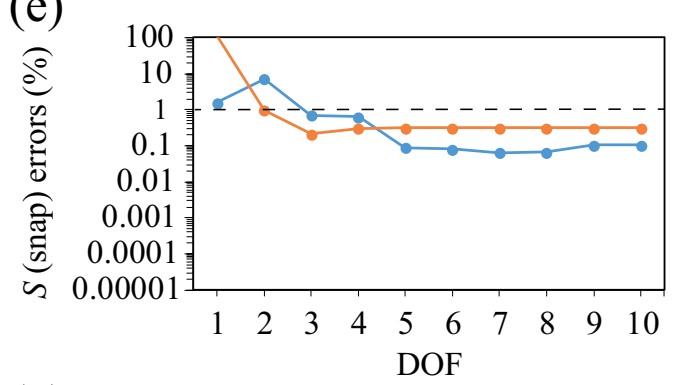

$(\mathrm{g})$

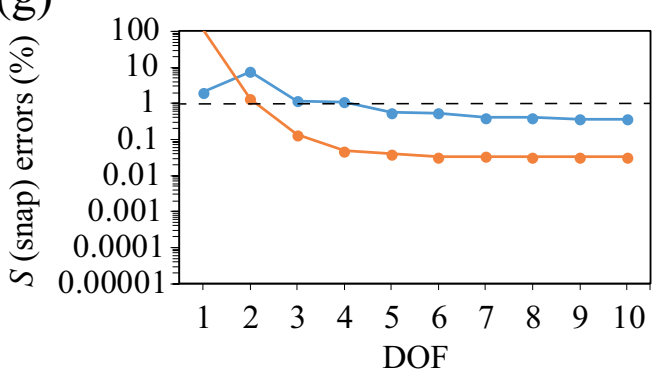

(b)

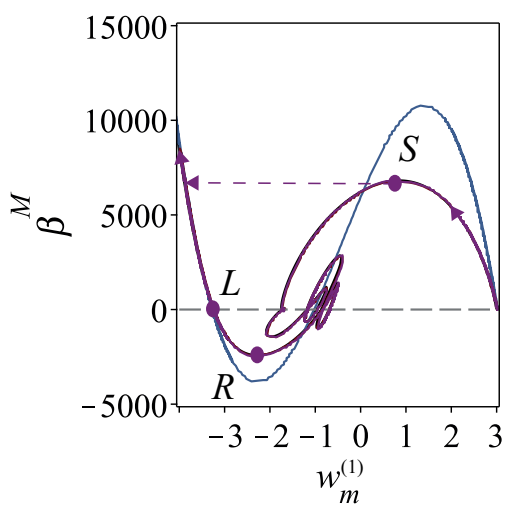

(d)

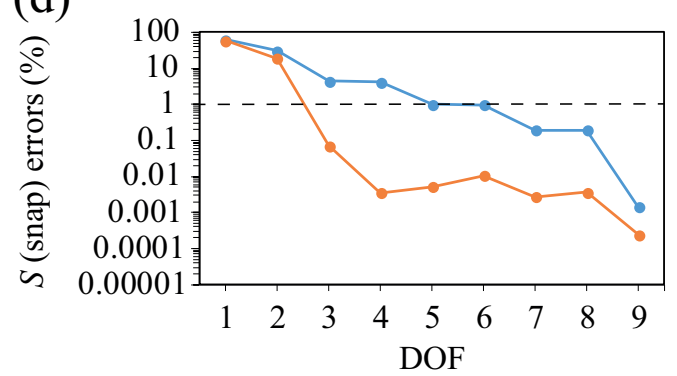

(f)

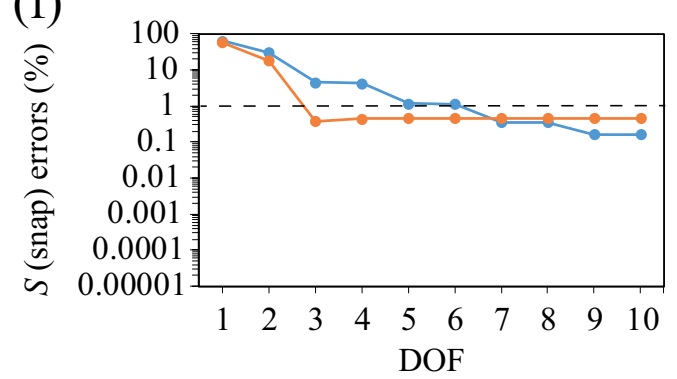

(h)

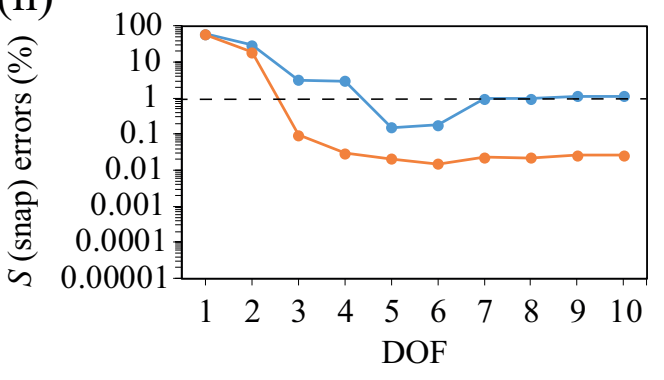

Figure 2: Equilibrium curves of mechanically loaded double beam structures showing the load $\beta^{M}$ as a function of the midpoint location of the Driving Beam, $w_{m}^{(1)}$, for two configurations: (a) $h_{0}^{(1)}=4, h_{0}^{(2)} / h_{0}^{(1)}=$ 0.7 (b) $h_{0}^{(1)}=3, h_{0}^{(2)} / h_{0}^{(1)}=1.3$. Blue, green and red lines represent single, three and five DOF RO model results, while purple and black represent results obtained via a direct FD solution and FE model, respectively. Grey dashed line signifies $\beta^{M}=0$. Points $S, R$ and $L$ represent the snap-through, release and latching points, respectively, corresponding to the curve obtained via FE. Arrows signify the beam midpoint movement along the equilibrium curve to eventual snap-through and release (when relevant), according to the FE model. The following error curves are given as a function of DOF/mode number $(n)$, with blue and orange lines representing the snap-through location and load errors, respectively: (c)(e)(g) With respect to ten DOF RO model, FD solution and FE model, corresponding to the case given in (a). (d)(f)(g) With respect to ten DOF RO model, FD and FE, corresponding to the case given in (b). Black horizontal dashed lines represent the $1 \%$ error threshold. 
connected. The reaction force is thus found from the midpoint node of Beam \#2 and placed in the corresponding equation of Beam \#1. From here, a change of variable is carried out as was done for the RO model, while ensuring that the displacement of the two beams is equal, creating $4 N-4$ DOF, where $N$ is the number of total segments. For the sake of a reliable solution, FD solutions were carried out for $N=100$ segments, following a convergence study that provided an error smaller than one percent in snap-through location and load for a structure having $h_{0}^{(1)}=3, h_{0}^{(2)} / h_{0}^{(1)}=0.5$, when compared to $N=50$.

With the establishment of the references, we move on to the case study. A comparison between the models under mechanical load is given in Figs. 2(a), (b), showing non-dimensional equilibrium plots per the definitions set out in Table 1. The curves were extracted from single, three and five DOF RO models, superimposed against FD and FE solutions, shown in blue, green, red, black and purple, respectively. The plots depict the dependence between the load $\left(\beta^{M}\right)$, against the midpoint location of the Driving Beam $\left(w_{m}^{(1)}\right)$, for two different cases. The first case, Fig. 2(a), shows a structure with a configuration of $h_{0}^{(1)}=4, h_{0}^{(2)} / h_{0}^{(1)}=0.7$, presenting bistability with both snap-through $(S)$ and release $(R)$ points. In the second case, Fig. 2(b), the curve demonstrates bistability with a latching point $(L)$, occurring when a negative release load $\left(\beta_{R}^{M}<0\right)$ is present $[33,34]$. Note that for this geometry, the Driving Beam exhibits a complicated unstable branch. Although that is to be expected from bistable structures such as single curved beams and plates with high enough curvatures, the pattern shown here is quite distinctive, suggesting that mode interaction is different when compared to single beams [34]. This behaviour is attributed to the fact that the Driving Beam has a different loading scheme, compared to single beams. The latter had only a lateral load, while the former has also a concentrated force at the midpoint, originating from the Secondary Beam.

With relation to the juxtaposition of all five plots, it is evident that from a visual perspective, a single DOF is not able to reliably represent an equilibrium curve, whilst the rest surprisingly overlap. To obtain a quantifiable comparison, we turn to the convergence plots. Figures 2(c), (e), (g) show the convergence of the snap-through location and load for the 
case given in Fig. 2(a) with respect to ten DOF RO, FD and FE models, respectively. From the trend given against ten DOF model, convergence is evident, however when comparing to $\mathrm{FD}$, one can see that the RO model reaches convergence at $n=5$ for the location, and $n=3$ for the load, with errors of $\approx 0.08 \%$ and $\approx 0.21 \%$, respectively. Looking at the comparison to FE, a similar trend of convergence is granted again, with saturation at $n=5$ for both location and load, and errors of $\approx 0.56 \%$ and $\approx 0.04 \%$, respectively. Looking at the second case, Fig. 2(b), with its corresponding convergence plots in Figs. 2(d), (f), (g), we witness a similar trend. Figure 2(d) shows a steady convergence to ten DOF model, while Fig. 2(f) presents saturation at $n=3$ for the snap-through load with a value of $\approx 0.38 \%$, and a slow convergence for the location when comparing to FD. When taking a closer look at the location, we see that it reaches an error of $\approx 1.16 \%$ at $n=5$, while the curve passes below one percent error at $n=7$ to $\approx 0.36 \%$. When comparing against FE, a somewhat similar behaviour is given. The curve saturates at $n=5$ with an error of $\approx 0.02 \%$ for the load, and $n=7$ with $\approx 0.95 \%$ for the location, while crossing the one percent threshold at $n=5$ to $\approx 0.15 \%$.

Note that in order to model the two structures using FE, different gaps (i.e., $\left|w^{(1)}(0)-w^{(2)}(0)\right|$ ) had to be used due to different elevation ratios. Specifically, a gap of $10 \mu \mathrm{m}$ was used for the case in Fig. 2(a), while a gap of $15 \mu \mathrm{m}$ had to be taken for the case in Fig. 2(b). Although different gaps were used in the modelling process, it did not hamper the overlap in equilibrium curves.

From these comparisons it is possible to conclude that for this type of structure, a minimum of three DOF are needed to attain a qualitative equilibrium curve, and five DOF for a quantitatively satisfactory one, having errors below one percent.

\subsection{Electrostatic load}

With the establishment of the RO model for the mechanical load, we proceed to the electrostatic case. To do so, we substituted $\mathbf{f}=\mathbf{f}^{e}$, as defined in Eq. (14), in Eq. (12) for the RO model, and $f=f^{e}$ from Eq. (9), in Eq. (7) for the FD solution. For the latter, Eq. (7)

was multiplied by $\left(1+w^{(1)}\right)^{2}$ for Beam $\# 1$ to facilitate its solution. As for the RO model, 
(a)

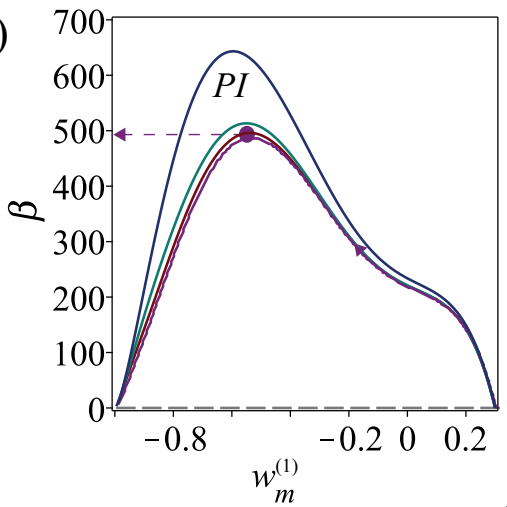

(d)

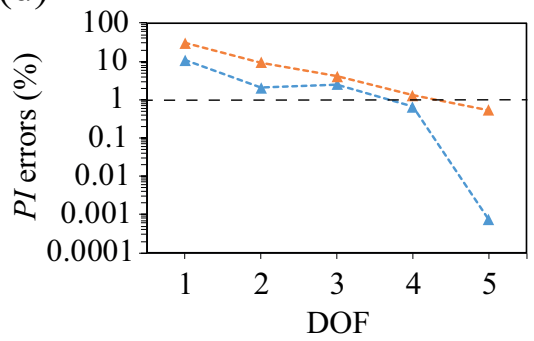

(g)

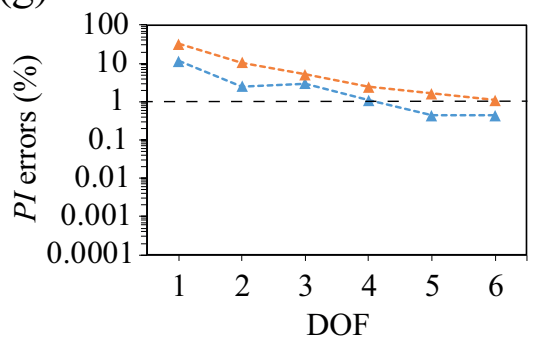

(b)

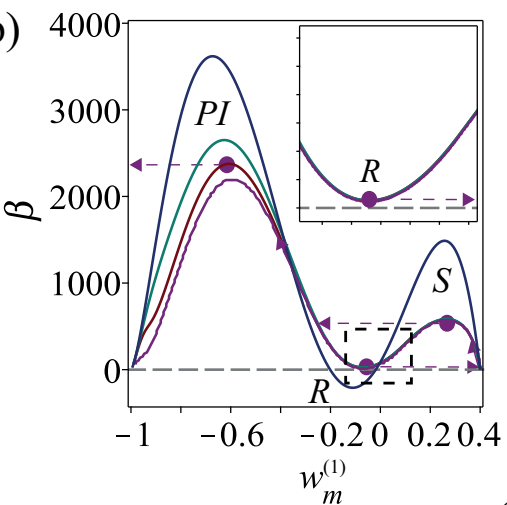

(e)

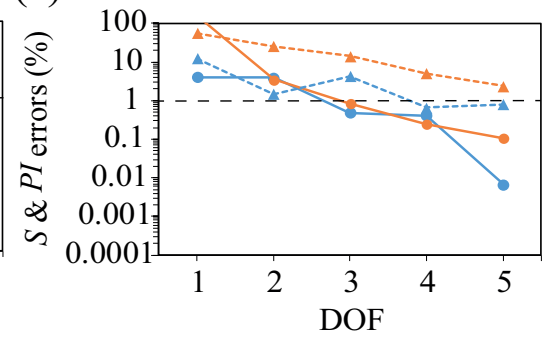

(h)

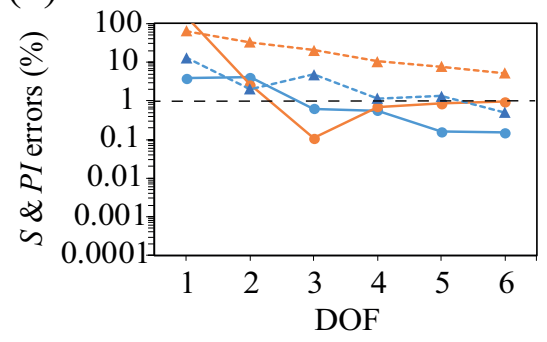

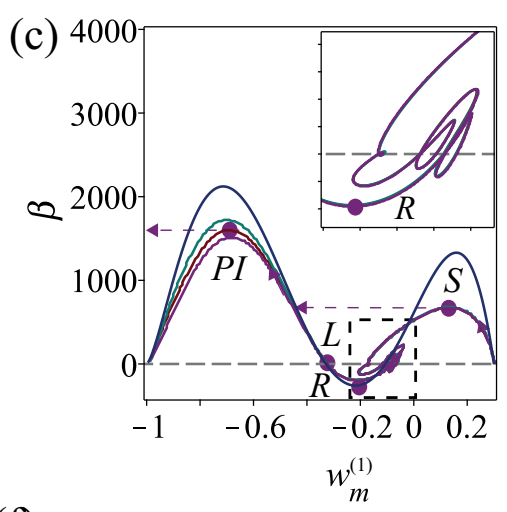

(f)

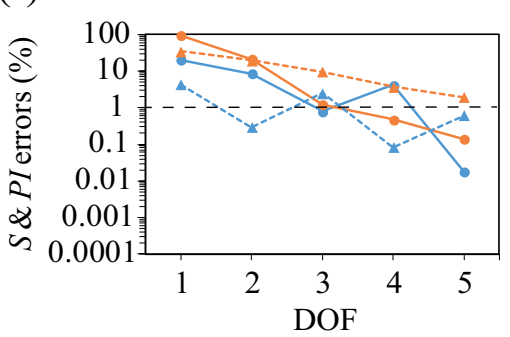

(i)

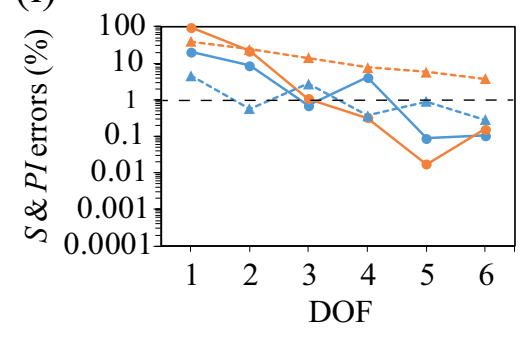

Figure 3: Equilibrium curves of electrostatically loaded double beam structures, with three configurations: (a) $d=0.3, h_{0}^{(1)}=0.3, h_{0}^{(2)} / h_{0}^{(1)}=0.5$ (b) $d=0.1, h_{0}^{(1)}=0.4, h_{0}^{(2)} / h_{0}^{(1)}=0.5$. (c) $d=0.1, h_{0}^{(1)}=0.3$, $h_{0}^{(2)} / h_{0}^{(1)}=1.3$. Inserts, given in (b) \& (c) provide closeups at the vicinity of the release point, corresponding to the dashed black rectangles. Blue, green, red and purple lines represent single, three, six DOF RO models and FD solution, respectively. The grey dashed line represents $\beta=0$. Points $S, R, L$ and $P I$ represent the snap-through, release latching and pull-in points, respectively, as predicted by the FD solution. Arrows signify the beam midpoint movement along the equilibrium curve to eventual snap-through, pull-in and release (when relevant), per the FD solution prediction. The following error curves are given as a function of mode/DOF number $(n)$. Solid with circular points, and dashed curves with triangular points represent snap-through $(S)$ and pull-in $(P I)$ error curves, respectively. Blue and orange lines signify location and load, respectively: (d)(e) Errors with respect to five DOF RO model and FD solution, respectively, corresponding to case (a). (e)(h) Errors with respect to five DOF RO model, and FD solution, respectively, corresponding to case (b). (f)(i) Errors with respect to five DOF RO model, and FD solution, respectively, corresponding to case (c). Black horizontal dashed lines represent the $1 \%$ error threshold. 
the integral that depicts the load was solved numerically using adaptive Gauss 30-point and Kronrod 61-point rules [56]. Since the loading is different, so does the normalization, as given in Table 2. Also note, that similar to the previous case study, we track the location of the Driving Beam, $w^{(1)}$. However, in contrast to the mechanical case, the Secondary Beam is now located above the Driving Beam and is being pulled, rather than pushed, as seen in Fig. 1(b).

Looking at Fig. 3, three cases are shown. The case in $(\mathrm{a})$, having $d=0.3, h_{0}^{(1)}=0.3$, $h_{0}^{(2)} / h_{0}^{(1)}=0.5$, shows a monostable structure with one limit point, the pull-in $(P I)$. The case in (b), with $d=0.1, h_{0}^{(1)}=0.4, h_{0}^{(2)} / h_{0}^{(1)}=0.5$, exhibits bistability with both snap-through and pull-in $(S \& P I)$, and case $(\mathrm{c})$, having $d=0.1, h_{0}^{(1)}=0.3, h_{0}^{(2)} / h_{0}^{(1)}=1.3$, presents a bistable structure with swerving non-stable equilibrium curve, as well as a latching point $(L)$, similar to what was observed in Fig. 2(b). Every case presents curves extracted via single, three, and five DOF RO models, as well as an FD based solution in blue, green, red and purple, respectively. From a visual perspective, it seems that one DOF is not reliable once again, even when the structure is monostable, although some overlap exists closer to the initial location, $h_{0}^{(1)}$. Such observation holds true for the rest as well, since they seem to overlap close to the initial configuration, while getting further apart as the curves approach the electrode, located at $w_{m}^{(1)}=-1$. Moreover, since higher modes are present in cases (b) and especially case (c), three DOF seems to be a minimum required for a qualitative equilibrium curve, as was also noticeable for the mechanical load.

The result of the convergence studies are given in Figs. 3(d), (g) for case (a); Figs. 3(e), (h) for case (b), and Figs. 3(f), (i) for case (c) against five DOF and FD solutions, respectively, for each case. In addition to the snap-through location and load error values, shown as solid lines and circular points, we also observed the pull-in point, represented by triangle points and dashed lines. Blue and orange lines represent, as before, location and load error curves, respectively. Observing the convergence with respect to six DOF, it is possible to notice that the RO model can converge, albeit at different rates, especially when comparing the trends of the snap-through and pull-in points. Observing the errors 
attained for case (a), five DOF once again looks as a minimum number of modes required for a reasonable error, achieving error percentages of $\approx 0.44 \%$ and $\approx 1.65 \%$ for the pull-in location and load, respectively. Refocusing our attention to the convergence in (g)-(i) with respect to FD, we witness a "slower" convergence of the pull-in curves relative to the snapthrough, while attaining a smaller error in location with resect to the load, with some of the curves achieving an error below one percent at five DOF, such as case (b). This is attributed to high axial forces, present when the beam is closer to the electrode, requiring a large number of modes in the calculation. Such attributions correspond to the visual comparisons made earlier, where it was noticeable that the curves differ themselves from one another the closer they got to the electrode. It is important to point out, that such distinction was previously made in [53] for curved plates, showing a similarity to a complicated bistable structure. As for the snap-through, case (b) crosses the one percent threshold at $n=3$ with an error of $\approx 0.63 \%$ for the location, and $\approx 0.11 \%$ for the load. Case $(\mathrm{c})$ makes the same transition with errors of $\approx 0.79 \%$ for the location at $n=3$ and $\approx 0.48 \%$ for the load at $n=4$.

From the above it is possible to conclude that for a reliable quantitative response, with errors lower than one percent, five DOF are needed. Such a distinction once again foretells that a relatively large number of modes are interacting with each other, especially when comparing the current structure to a single curved beam.

It is curios to point out that such observations were previously made regarding curved axisymmetric shallow curved plate models $[53,57]$, which due to their complexity and mode interaction, required a similar number of modes. Such a conclusion reflects on the curves given here, especially the one in Fig. 2(b), 3(c), that together with the convergence patterns show that a relativity large number of modes are in play. In contrast, single RO models proved to be of value in the case of bistable shallow beams, which repeatedly proved their reliability $[25,44,48]$. 

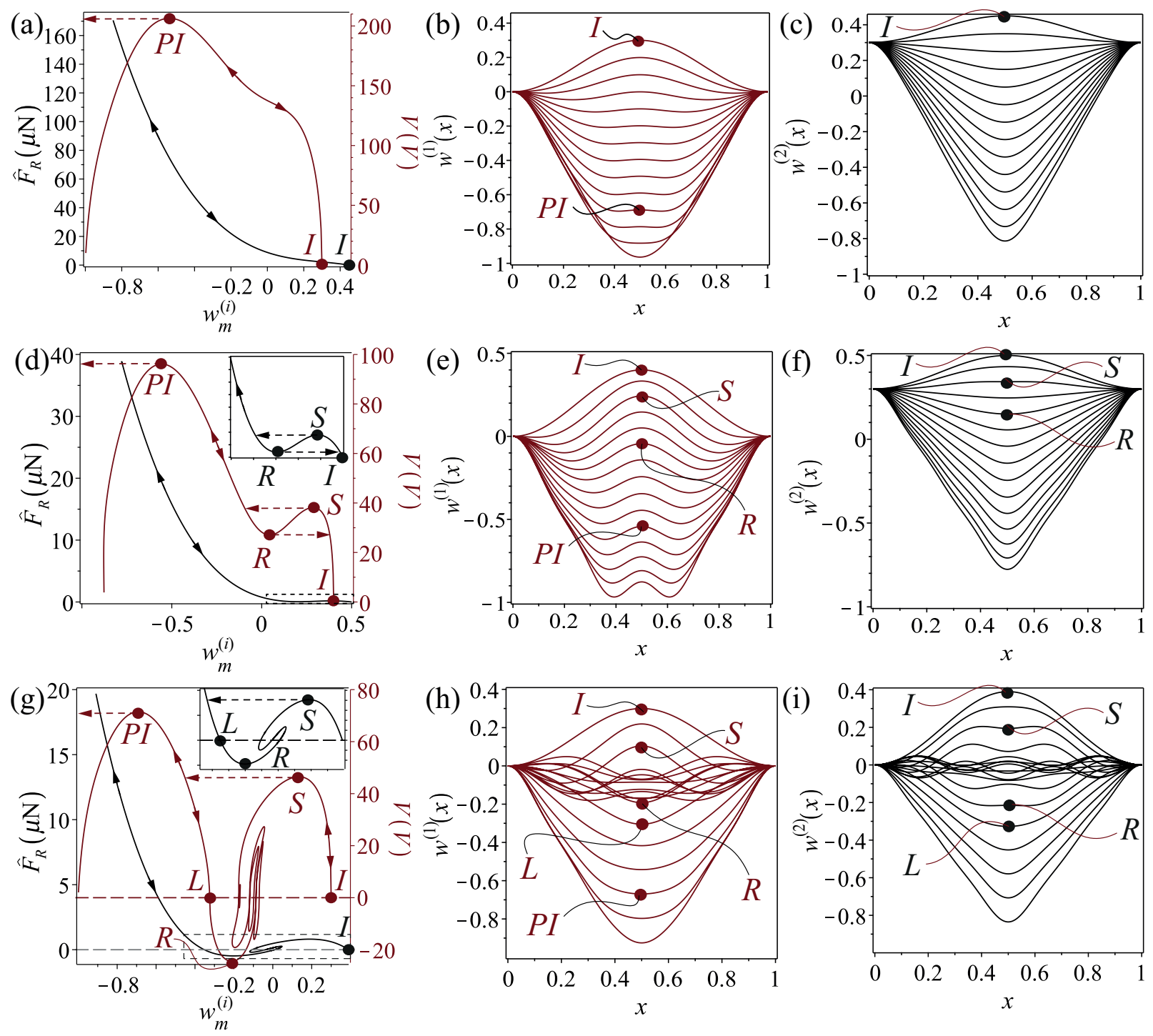

Figure 4: Dimensional equilibrium curves of electrostatically actuated double beam structures, calculated using a five DOF RO model. The plots show the dependency between the voltage $(V)$ acting on the Driving Beam $(i=1)$ in red, and the reaction force $\left(\hat{F}_{R}\right)$ acting on the secondary Beam $(i=2)$, in black, as a function of the nondimensional midpoint location of the $i^{\text {th }}$ beam, $w_{m}^{(i)}$. (a) $\hat{d}=3 \mu \mathrm{m}, \hat{h}_{0}^{(1)}=3 \mu \mathrm{m}, \hat{h}_{0}^{(2)}=1.5 \mu \mathrm{m}$, with $V_{P I} \approx 205.6 \mathrm{~V}$. The plots correspond to visualizations given in (b) \& (c) for Beam \#1 and \#2, respectively. (d) $\hat{d}=1 \mu \mathrm{m}, \hat{h}_{0}^{(1)}=4 \mu \mathrm{m}, \hat{h}_{0}^{(2)}=3.9 \mu \mathrm{m}$, with $V_{S} \approx 38.04 \mathrm{~V}, V_{R} \approx 26.99$, and $V_{P I} \approx 95.78 \mathrm{~V}$. The plots correspond to beam visualizations seen in (e) \& (f) for Beam \#1 and \#2, respectively. (g) $\hat{d}=1 \mu \mathrm{m}$, $\hat{h}_{0}^{(1)}=3 \mu \mathrm{m}, \hat{h}_{0}^{(2)}=3.9 \mu \mathrm{m}$, with $V_{S} \approx 46.28 \mathrm{~V}, V_{R} \approx-24.47 \mathrm{~V}$, and $V_{P I} \approx 71.14 \mathrm{~V}$. The plots correspond to beam visualizations given in (h) \& (i) for Beam \#1 and \#2, respectively. All beams have a width of $b=20 \mu \mathrm{m}$, Young's modules $\tilde{E}=169 \mathrm{GPa}$, and length of $L=1000 \mu \mathrm{m}$, with a gap of $g_{0}=10 \mu \mathrm{m}$. Points $I$, $S, R, L$ and $P I$ represent the initial location, snap-through, release, latching and pull-in points, respectively. Red and black dashed lines represent $V=0$, and $\hat{F}_{R}=0$, respectively. Dashed rectangles in (d) \& (g) show closeups of the Secondary Beam equilibrium curve at the vicinity of the the snap-through and release points. 


\section{Dimensional example}

With the validation of the model, and following the conclusions made in the previous sections, we focus our attention to dimensional examples, solved using a five DOF RO model. In doing so we hope to gain further insight into the interaction between the two beams, through their equilibrium curves, as well as corresponding visualizations. To attain a solution for the Secondary Beam, we remember that the arc-length method algorithm practically solves both simultaneously, since the general coordinates of Beam \#2 are considered as additional DOF in the system. Therefore, all that remains is to isolate the variables that represent Beam \#2 (Sec. 2.1) at each step, and substitute them back to one of the equations describing the Secondary Beam, Eq. (12) $(i=2)$. Making it so will enable us to attain a solution for the concentrated force $F_{R}^{e}$ at each increment. The result of this procedure is given in Fig. 4, showing three cases, two of which correspond to the non-dimensional electrostatic case study from Fig. 3, with $d=1 \mu \mathrm{m}$ and $g_{0}=10 \mu \mathrm{m}$. Specifically, Figs. 4(a), (c) correspond to Figs. 3(a), (c). To disclose their interaction, we juxtapose both equilibrium curves on the same plot, with red representing Beam \#1 and black, Beam \#2. Corresponding to each equilibrium response, are two visualisations, showing each beam in red and black per the same identification, while marking different points of interest, $I, S, R$, $L$ and $P I$, with $I$ being the initial configuration, $w_{I}^{(1)} \triangleq h_{0}^{(1)} \& w_{I}^{(2)} \triangleq h_{0}^{(2)}$.

From an overall view of the results, we note that when one beam is bistable, so is the other, as expected from the compatibility condition, Eq. (3). However, when diving into the exact nature of each curve, and its visual counterpart, new details emerge. We first look at Fig. 4(a), demonstrating a monostable curve with a pull-in (PI) response for Beam \#1. Observing its corresponding visualization, Fig. 4(b), we notice that contrary to what one expects from a pull-in reaction, the midpoint does not lean towards the electrode [21]. The reason for this is Beam \#2, located above Beam \#1, pulling it upwards during actuation, creating a larger reaction force as the Driving Beam gets closer to the electrode. As for the bistable configuration in Fig. 4(d), we see a more extreme case of the pull-in, causing Beam \#1 to collapse to the electrode due to a shape which is a superposition of several modes. 
The same observation is valid for the release point $(R)$, showing a more complicated shape than the one present in Beam \#2. This foretells that higher modes, usually not encountered in single curved beams, take a significant role with relativity higher amplitudes, even though the equilibrium curve of Beam \#1 does not show it at first glance. Indeed, by extracting the values of the general coordinates, it is possible to witness that while the first mode is the most dominant, followed by the second, the fourth mode has a slightly higher amplitude than the third. Note, that due to this behaviour, the resulting curve ends sooner than expected, which shows the equilibrium curve at the midpoint. All this occurs while Beam \#2 does not show unusual behaviour in its visualization, remaining true to what one would assume from a curved beam under a concentrated mechanical load. Regarding the case in Fig. 4(g), we notice that the unstable curves of the beams differ from one another, with corresponding different visualizations. Beam \#1 shows complicated loops as was previously shown in its non-dimensional counterpart, Fig. 3(c), while Beam \#2 presents a "simpler" curve. Noticing that, it is possible to tell that each beam uses different modes in its overall reaction once again, while being more pronounced. An observation which is corroborated in the visualizations, especially at the unstable regions (between $S$ and $R$ ), as well as the one depicting the release point itself. Specifically, by observing the amplitudes of the general coordinates, it is possible to witness excitation of all modes in Beam \#1, while Beam \#2 manifests only the odd numbered ones.

Another conclusion that could be made for the dimensional case study, is that for the given set of parameters, such a structure could be actuated by relatively reasonable voltages, with a snap-through voltage at around $40 \mathrm{~V}$.

\section{Summary \& conclusions}

In this paper, we presented a case study and validation of an $\mathrm{RO}$ model, derived for a structure composed of two initially curved, nonidentical beams, coupled at their midpoints by a truss. The model was solved using the "Riks" method, which enabled proper tracking of swerving equilibrium curves, caused due to interaction of several modes. Since a rigid rod 
could have length and width that will limit both beams to symmetric modes, we focused the current study to the analysis of the symmetric response.

The model was first verified for mechanical, displacement-independent load, against FD and FE obtained solutions, with the latter serving as the reference. The validation process showed that the model can attain errors lower than one percent, when enough DOF are taken into account. Following that, the process continued to case study and validation under electrostatic, displacement-dependent load, using FD solutions as the reference. The study provided again that small errors can be achieved when enough DOF are brought into the fold, as was observed for the mechanical load. Specifically, it was deduced for both loads that at least three DOF are needed to get a qualitative response, and a minimum of five DOF are needed for a quantitative one. Therefore, it is possible to conclude that a double beam structure is far more complex than a single double clamped beam. Curiously enough, these observations were also made for curved plates, suggesting that a structure, composed of two one-dimensional beams, can be comparable in complexity to a two-dimensional axisymmetric curved plate.

Regarding the behaviour of the structure, it was observed that the Driving Beam, which directly encounters the load, can attain higher modes during its actuation with respect to the Secondary Beam. This creates a somewhat unique response, since either beam will present different mode interaction, simultaneously. The reason for the difference lies in the loading scheme that each beam faces. While the Secondary Beam responds to a reaction force emanating from the rigid rod, the Driving Beam responds to a lateral load, with an additional concentrated force at its midpoint. Furthermore, it was found through a dimensional case study that the structure can be actuated using realistic voltages.

To conclude, the derived RO model has shown that it is able to provide reliable equilibrium curves for several cases, monostable and bistable alike. As such, the model could be used to estimate the symmetric branches of such structures under electrostatic load, for a variety of applications, provided that the symmetric response is the dominating one.

Acknowledgments. L.M. acknowledges the support of the Blavatnik Postdoctoral Re- 
search fellowship. 


\section{References}

[1] J. Thompson, G. Hunt, A General Theory of Elastic Stability, Wiley-Interscience publication, J. Wiley, 1973.

[2] A. Frangi, B. De Masi, F. Confalonieri, L. Baldasarre, Threshold shock sensor based on a bi-stable mechanism, in: 2013 Transducers \& Eurosensors XXVII: The 17th International Conference on Solid-State Sensors, Actuators and Microsystems (TRANSDUCERS \& EUROSENSORS XXVII), IEEE, 2013, pp. 626-629.

[3] A. Frangi, B. De Masi, L. Baldasarre, Shock sensor with bistable mechanism and method of shock detection, 2016. US Patent 9,316,550.

[4] B. Hansen, C. Carron, B. Jensen, A. Hawkins, S. Schultz, Plastic latching accelerometer based on bistable compliant mechanisms, Smart Materials and Structures 16 (2007) 1967.

[5] J. Zhao, J. Jia, H. Wang, W. Li, A novel threshold accelerometer with postbuckling structures for airbag restraint systems, IEEE Sensors Journal 7 (2007) 1102.

[6] J. Zhao, Y. Yang, K. Fan, P. Hu, H. Wang, A bistable threshold accelerometer with fully compliant clamped-clamped mechanism, IEEE Sensors Journal 10 (2010) 1019-1024.

[7] S. Ilyas, M. A. Hafiz, S. Ahmed, H. Fariborzi, M. I. Younis, Toward cascadable microelectromechanical resonator logic units based on second vibration modes, AIP Advances 8 (2018) 105126.

[8] S. Ilyas, M. I. Younis, Resonator-based $\mathrm{m} /$ nems logic devices: Review of recent advances, Sensors and Actuators A: Physical (2019) 111821.

[9] S. Ilyas, M. A. Al Hafiz, H. Fariborzi, M. I. Younis, Mechanical resonator based cascadable logic device, 2020. US Patent 10,560,098.

[10] R. Popovic, K. Solt, H. Lienhard, Non-volatile memory device including a micromechanical storage element, 1990. US Patent 4,979,149. 
[11] B. Charlot, W. Sun, K. Yamashita, H. Fujita, H. Toshiyoshi, Bistable nanowire for micromechanical memory, Journal of Micromechanics and Microengineering 18 (2008) article number 045005 .

[12] M. A. García-Ramírez, A. Ghiass, Z. Moktadir, Y. Tsuchiya, H. Mizuta, Fabrication and characterisation of a double-clamped beam structure as a control gate for a high-speed non-volatile memory device, Microelectronic Engineering 114 (2014) 22-25.

[13] M. A. Al Hafiz, L. Kosuru, M. I. Younis, Electrothermal frequency modulated resonator for mechanical memory, Journal of Microelectromechanical Systems 25 (2016) 877-883.

[14] S. A. Zirbel, K. A. Tolman, B. P. Trease, L. L. Howell, Bistable mechanisms for space applications, PloS one 11 (2016).

[15] H. Du, F. S. Chau, G. Zhou, Harmonically-driven snapping of a micromachined bistable mechanism with ultra-small actuation stroke, Journal of Microelectromechanical Systems 27 (2018) 34-39.

[16] R. L. Harne, K. Wang, A review of the recent research on vibration energy harvesting via bistable systems, Smart materials and structures 22 (2013) 023001.

[17] N. Tran, M. H. Ghayesh, M. Arjomandi, Ambient vibration energy harvesters: A review on nonlinear techniques for performance enhancement, International Journal of Engineering Science 127 (2018) 162-185.

[18] A. J. Lee, D. J. Inman, Electromechanical modelling of a bistable plate with macro fiber composites under nonlinear vibrations, Journal of Sound and Vibration 446 (2019) 326342.

[19] G. J. Simitses, Dynamic Stability of Suddenly Loaded Sturctures, New York SpringerVerlag, 1989.

[20] G. J. Simitses, D. H. Hodges, Fundamentals of Structural Stability, ButterworthHeinemann, 2006. 
[21] W.-M. Zhang, H. Yan, Z.-K. Peng, G. Meng, Electrostatic pull-in instability in mems/nems: A review, Sensors and Actuators A: Physical (2014).

[22] E. Loukaides, S. Smoukov, K. Seffen, Magnetic actuation and transition shapes of a bistable spherical cap, International Journal of Smart and Nano Materials 5 (2014) $270-282$.

[23] K. Das, R. C. Batra, Pull-in and snap-through instabilities in transient deformations of microelectromechanical systems, Journal of Micromechanics And Microengineering 19 (2009) article number 035008.

[24] K. Das, R. C. Batra, Symmetry breaking, snap-through and pull-in instabilities under dynamic loading of microelectromechanical shallow arches, Smart Materials and Structures 18 (2009) article number 115008.

[25] S. Krylov, B. Ilic, D. Schreiber, S. Seretensky, H. Craighead, The pull-in behavior of electrostatically actuated bistable microstructures, Journal of Micromechanics and Microengineering 18 (2008) 055026.

[26] L. Medina, R. Gilat, S. Krylov, Bistable behavior of electrostatically actuated initially curved micro plate, Sensors and Actuators A: Physical 248 (2016) 193-198.

[27] M. H. Ghayesh, H. Farokhi, M. Amabili, Nonlinear behaviour of electrically actuated mems resonators, International Journal of Engineering Science 71 (2013) 137-155.

[28] R. Batra, M. Porfiri, D. Spinello, Reduced-order models for microelectromechanical rectangular and circular plates incorporating the casimir force, International Journal of Solids and Structures 45 (2008) 3558-3583.

[29] M. Vangbo, An analytical analysis of a compressed bistable buckled beam, Sensors and Actuators A: Physical 69 (1998) 212-216.

[30] J. Qiu, J. Lang, A. H. Slocum, A curved-beam bistable mechanism, Journal of Microelectromechanical Systems 13 (2004) 137-146. 
[31] J. Qiu, A. H. Slocum, J. H. Lang, R. Struempler, M. P. Brenner, J. Li, Bistable actuation techniques, mechanisms, and applications, 2005. US Patent 6,911,891.

[32] Y. Gerson, S. Krylov, B. Ilic, Electrothermal bistability tuning in a large displacement micro actuator, Journal of Micromechanics and Microengineering 20 (2010) 112001.

[33] L. Medina, R. Gilat, S. Krylov, Latching in bistable electrostatically actuated curved micro beams, International Journal of Engineering Science 110 (2017) 15 - 34.

[34] M. Nistor, R. Wiebe, I. Stanciulescu, Relationship between euler buckling and unstable equilibria of buckled beams, International Journal of Non-Linear Mechanics 95 (2017) $151-161$.

[35] W.-B. Kim, S.-Y. Han, Microinjection molding of out-of-plane bistable mechanisms, Micromachines 11 (2020) 155.

[36] L. Medina, R. Gilat, S. Krylov, Dynamic release condition in latched curved micro beams, Communications in Nonlinear Science and Numerical Simulation 73 (2019) 291-306.

[37] H.-W. Huang, Y.-J. Yang, A mems bistable device with push-on-push-off capability, Journal of microelectromechanical systems 22 (2012) 7-9.

[38] C.-C. Wu, M.-J. Lin, R. Chen, The derivation of a bistable criterion for double v-beam mechanisms, Journal of Micromechanics and Microengineering 23 (2013) 115005.

[39] D. Yadav, N. S. Murthy, S. Palathingal, S. Shekhar, M. Giridhar, G. Ananthasuresh, A two-terminal bistable electrothermally actuated microswitch, Journal of Microelectromechanical Systems 28 (2019) 540-549.

[40] H.-W. Huang, F.-W. Lee, Y.-J. J. Yang, Design criteria for a push-on push-off mems bistable device, Journal of Microelectromechanical Systems 25 (2016) 900-908. 
[41] H. M. Ouakad, Stability analysis of initially curved beams mechanically coupled in a parallel arrangement, International Journal of Structural Stability and Dynamics 18 (2018) 1850041.

[42] S.-W. Huang, F.-C. Lin, Y.-J. Yang, A novel bistable device driven by single actuator via moment input, in: 2020 IEEE 33rd International Conference on Micro Electro Mechanical Systems (MEMS), IEEE, 2020, pp. 618-621.

[43] M. H. LOCK, Snapping of a shallow sinusoidal arch under a step pressure load., AIAA Journal 4 (1966) 1249-1256.

[44] M. Younis, MEMS Linear and Nonlinear Statics and Dynamics, Microsystems Series, Springer, 2011.

[45] P. Villagio, Mathematical Models for Elastic Structures, Cambridge, Cambridge University Press, 1997.

[46] L. Medina, R. Gilat, B. Ilic, S. Krylov, Experimental investigation of the snap-through buckling of electrostatically actuated initially curved pre-stressed micro beams, Sensors and Actuators A: Physical 220 (2014) 323 - 332.

[47] S. Krylov, N. Dick, Dynamic stability of electrostatically actuated initially curved shallow micro beams, Continuum Mechanics and Thermodynamics 22 (2010) 445-468.

[48] L. Medina, R. Gilat, S. Krylov, Symmetry breaking in an initially curved micro beam loaded by a distributed electrostatic force, International Journal of Solids and Structures 49 (2012) $1864-1876$.

[49] L. Medina, R. Gilat, S. Krylov, Symmetry breaking in an initially curved pre-stressed micro beam loaded by a distributed electrostatic force, International Journal of Solids and Structures 51 (2014) 2047 - 2061. 
[50] L. Medina, R. Gilat, S. Krylov, Bistability criterion for electrostatically actuated initially curved micro plates, International Journal of Engineering Science 130 (2018) $75-92$.

[51] M. Crisfield, A fast incremental/iterative solution procedure that handles snap-through, Computers \& Structures 13 (1981) 55-62.

[52] J. Bonet, R. Wood, Nonlinear continuum mechanics for finite element analysis, Cambridge Univ Press, 1997.

[53] L. Medina, R. Gilat, S. Krylov, Modeling strategies of electrostatically actuated initially curved bistable micro plates, International Journal of Solids and Structures 118 (2017) $1-13$.

[54] Dassault Syst Ǎšmes, Abaqus 6.13 Online Documentation, http://50.16.176.52/v6. 13/, 2013.

[55] S. D. Conte, C. De Boor, Elementary Numerical Analysis: An Algorithmic Approach, McGraw-Hill Book Company, 1980.

[56] Maplesoft, a division of Waterloo Maple Inc., Numerical Integration, https: //www .maplesoft.com/support/help/Maple/view. aspx?path=evalf/Int\&term= evalf/int, 2020. Online software support.

[57] P. Sobota, K. A. Seffen, Effects of boundary conditions on bistable behaviour in axisymmetrical shallow shells, Proceedings of the Royal Society A: Mathematical, Physical and Engineering Sciences 473 (2017) 20170230. 